\title{
Development of Heartbeat Based Biometric System Using Wavelet Transform
}

\author{
Chin Chee Yeen and Dzati Athiar Ramli* \\ School of Electrical and Electronic Engineering, USM Engineering Campus, \\ Universiti Sains Malaysia, 14300 Nibong Tebal, Pulau Pinang, Malaysia \\ "Corresponding author:dzati@usm.my
}

Published online: 15 April 2018

To cite this article: Chin Chee Yeen and Dzati Athiar Ramli. (2018). Development of heartbeat based biometric system using wavelet transform. Journal of Engineering Science, 14: 15-33, https://doi.org/10.21315/jes2018.14.2.

To link to this article: https://doi.org/10.21315/jes2018.14.2

\begin{abstract}
Electrocardiogram (ECG) is a new biometric trait that has an advantage as it is an internal modality which indicates to be difficult of counterfeiting. The ECG records the heart activity and it is unique as different individuals have distinct heart structure. Hence, it provides vital information for differentiating one individual from another. One of the techniques to extract the salient information from the ECG signal is by using discrete wavelet transform (DWT). However, in order to develop a reliable ECG based biometric system using $D W T$, there are many parameters that need to be determined. In this study, we study the effect of different parameters used for de-noising orders, threshold levels and type of mother wavelets to the final authentication performance of our developed system. For the authentication stage, Support Vector Machine (SVM) is used as classifier. The system performance is evaluated based on Genuine Acceptance Rate (GAR), False Acceptance Rate (FAR) and Equal Error Rate (EER) by using the scores given by the SVM. The combination of de-noising order 3, heursure threshold method and db6 mother wavelet yields the top performance with GAR of $92.5 \%$ at FAR of $5 \%$ and EER of $6.9499 \%$ have been achieved. At the end of this paper, a simple ECG based security system is then constructed so as to show the feasibility of this study to be implemented in real-time situation.
\end{abstract}

Keywords: Biometrics, electrocardiogram, wavelet transform, authentication, support vector machine

\section{INTRODUCTION}

Accurate and reliable authentication systems are very crucial nowadays because frauds and disclosure of personal data can lead to a major harm. Traditional authentication methods such as password and token which are among the oldest authentication methods prone to problems of loss and stolen. ${ }^{1}$ Biometric 
recognition is science that uniquely identifies individual by means of his or her physiological or behavioural characteristics. In this computer-driven era, it is mostly used to tackle problems of assessing control to the security system. However, the existing biometric identifiers based on biological traits: fingerprint, face, palm print or behavioural characteristics: gait, signature, typing pattern are not that reliable as they can also be counterfeited. ${ }^{2}$

In medical field, electrocardiogram (ECG) is commonly used to detect cardiovascular diseases. Heartbeat is a reflection of the mechanical movement of the heart and cardiovascular system and these traits indicate heartbeat contains physiological and pathological information. Recently, researchers conclude that humans' electrocardiogram (ECG or EKG) are unique to all individual as different individuals have different heart structure. Hence, this has attracted several researches to turn to the study of ECG modality for biometric system., ${ }^{3,4}$ The problems of counterfeiting the original biometric data such as by using recording speech, fake gummy palm/finger print and captured photo; can be overcome with ECG. ${ }^{3}$ This is because heartbeat is a promising biometric characteristic because it cannot be faked easily as it involves complex biological process to produce it. On the other hand, in term of universality, it can be used broadly in areas that require security access, such as door entry, mobile phone, and laptop access.

The basic patterns of electrical activity produced by the heart consist of $P, Q R S$ and $T$ waves as shown in Figure 1. The $P$ wave, representing atrial depolarization and it corresponds to low frequency spectral components of 10 to $15 \mathrm{~Hz}$. The $Q R S$ complex indicates ventricular depolarization and contraction. It has much steeper slopes compared to other waves and it occurs in 10 to $40 \mathrm{~Hz}$ interval. The amplitude of $Q R S$ complex is generally greater than the $P$ wave. The $P R$ segment represents the electrical signal travelling from the sinus node to the heart ventricle. The $T$ wave has smaller amplitude than $Q R S$ complex and it is an upward waveform that indicates ventricular repolarization. ${ }^{5}$ Its position depends on the heart rate. When the heart rate is higher, the $T$ wave is closer to the $Q R S$ complex..$^{6,7}$

One of the techniques to extract the salient information from the ECG signal is by using discrete wavelet transform (DWT). However, in order to develop a reliable ECG based biometric system using DWT, there are many parameters that need to be determined. The main objective of this study is to investigate the effect of different parameters used for de-noising orders, threshold levels and type of mother wavelets to the final authentication performance of our developed system. In the next section, related works will be discussed in Section 2, followed by methodology in Section 3 and results and discussion in Section 4. Finally, Section 5 will give the conclusion. 


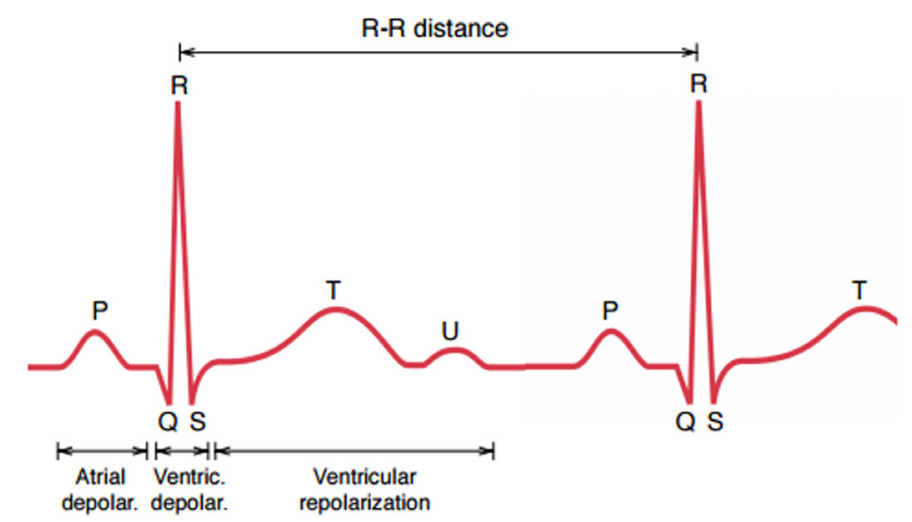

Figure 1: A typical normal ECG pulse.

\section{RELATED WORKS}

In research done by Kyoso and Uchiyama, ${ }^{8}$ an ECG identification system which identifies subjects by comparing a subject's ECG with registered ECG parameters in the database was developed. The first part of the research is the ECG measurement block where the durations and intervals of the ECG are measured. The four useful parameters are $P$ wave duration, $P Q$ interval, $Q R S$ interval and $Q T$ interval. The second block is the feature extraction block which extracts the four parameters from sampled ECG data sequence by placing characteristic points on the ECG. Second order derivative waveform is employed so that the precise location of the characteristic points can be located. Mahalanobis' generalised distance is employed for identification purpose. Lastly, two sequences within the useful range are chosen to enter the discriminant analysis block where the identification result is obtained. The combination of $Q R S$ interval and $Q T$ interval parameters provide the highest accuracy of $99.6 \%$.

Plataniotis et al. ${ }^{9}$ proposed a new approach by introducing Autocorrelation (AC) and Discrete Cosine Transform (DCT) for ECG biometrics. This method is called AC/DCT method. First, the ECG records go through filtering process by using pass-band filtering $(0.5$ to $40 \mathrm{~Hz})$ to remove noise and artifacts. Next, the correlation coefficients are calculated by applying normalised autocorrelation function on each record over an ECG window of $M$ autocorrelation lags. The performance of these two autocorrelation functions is evaluated using the normalised Euclidean and normalised Gaussian log likelihood. The third step is to calculate the DCT of the windowed correlation and the first C-number of significant coefficients are chosen. In the final step, the chosen DCT coefficients of two records are used for biometric identification. The best result obtained is a 
perfect subject recognition (14/14 subject recognition rate) using the Gaussian log likelihood function.

Saechia et al. $^{10}$ employed the normalisation of ECG signals for experimentation. Fourier Transform is employed for feature extraction. One period of $P Q R S T$ signal is divided into subsequences which correspond to $P, Q R S$ and $T$ waves respectively. Then, Fourier Transform is applied on PQRST period and also the three subsequences. Only the significant Fourier coefficients are used in the classification stage. The significant Fourier coefficients from whole period and subsequence cases are separately trained by neural network. The classification results were observed as $17.14 \%$ and $2.85 \%$ false rate (FR) using whole period of normalised ECG and subsequences of normalised ECG respectively.

Subsequently, research that uses the discrete wavelet transform (DWT) to extract the feature vectors from ECG signal was done by Belgacem. ${ }^{11}$ The mother wavelet used is Daubechies wavelet order 3 (DB3) with five level of decompositions. For experimentation, 80 healthy individuals' ECG signals obtained from the PhysioNet database were used. This study employed Random Forest technique as classifier. Consequently, the prototype of ECG sensor was developed by Ramli et al. ${ }^{12}$ in order to collect the ECG data in real-time condition. This study implemented wavelet transform as feature extraction by employing Symlet, Daubechies and Coiflet to evaluate the performance of the developed sensor. The normal ECG signals were collected from 50 individuals using the built heartbeat detection kit and this study achieved an EER of $2.0069 \%$ with GAR of $97 \%$ and FAR of $1 \%$.

\section{MATERIALS AND METHODS}

The ECG database used in this research is obtained from public available database, Physionet ECG-ID database. ${ }^{13}$ The total number of subjects in this database is 90 subjects with 310 numbers of ECG recordings, however only 79 subjects are selected for this study. The number of recordings for each subject varies from 2 to 20 and each recording contains ECG lead 1, recorded for $20 \mathrm{sec}$ and digitised at $500 \mathrm{~Hz}$. The main objective of this study is to investigate the effect of different parameters used for de-noising orders, threshold levels and type of mother wavelets to the final authentication performance of our developed system. In order to do this, we first need to develop a complete heartbeat based biometric system which consists of four modules i.e. pre-processing for denoising, segmentation, feature extraction and authentication (recognition) stages. The front-end developed system proposed in this study is shown in Figure 2. 


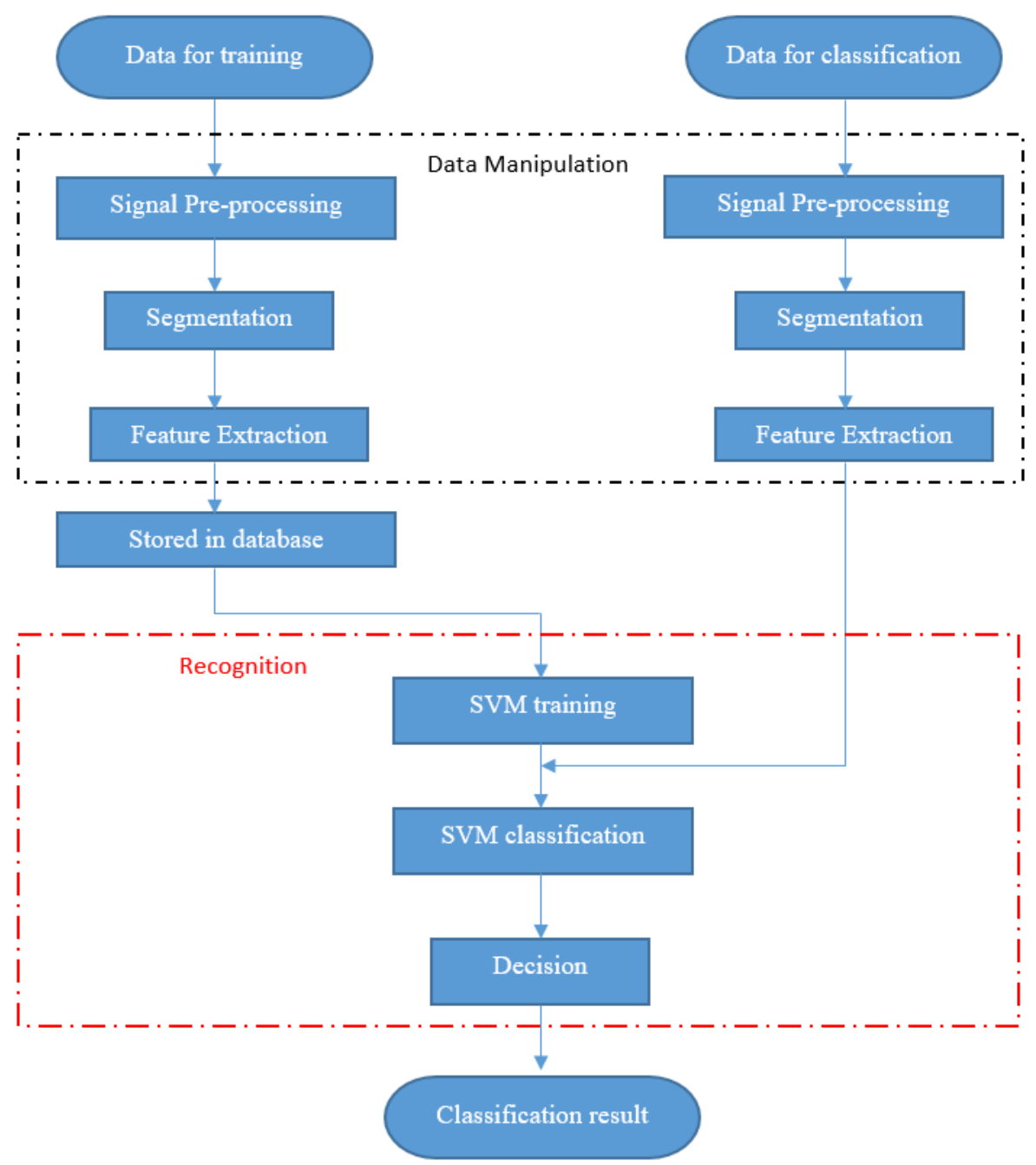

Figure 2: Overview of proposed biometric system.

In the experimentation process, the parameter for one variable will be changed while keeping the rest as constants, then the performance of the authentication system at the specified parameters will be recorded. Here, the parameters to be changed are de-noising orders, threshold levels and type of mother wavelets. By observing the experimental results, the optimum parameter for denoising order, threshold level and type of mother wavelet can be determined. Thus, the best ECG biometric system can be developed for the real time application. Finally, a simple ECG based security system will be constructed so as to show the feasibility of this study to be implemented for real-time authentication. 


\subsection{ECG Data Pre-Processing: Wavelet De-Noising Order and Threshold Level}

Two types of wavelet de-noising are performed in this process. First, the denoised algorithm is used for detection of $R$ peak and the second task of de-noising process is employed for feature extraction. The chosen parameters to be used in the first de-noised signal must yield ECG signals that show obvious $R$ peak while reducing other amplitude of $P$ and $T$ wave. For the second de-noised signal, the chosen parameters must produce an ECG signal that maintains the pattern which is similar to the raw ECG signal but with reduced noise and smoother signal. ${ }^{14}$

Following the standard practice, before the de-noising taking place, signal will first go through the de-trended process in order to remove the baseline wander noise. In order to do this, the signal is decomposed in eight levels using $d b 6$ wavelets. The low frequency components $\mathrm{D} 8$ and $\mathrm{A} 8$ have to be removed to obtain a baseline-free signal.

$$
\text { De-trended signal }=\text { Original signal }-(\text { A } 8+\text { D8 })
$$

Wavelet de-noising is then employed to remove the noise present in the signal. It concentrates the signal features in a few wavelet coefficients while removing noise. Generally, the de-noising process consists of three steps as shown in Figure 3:

1. L-Level Wavelet Decomposition of input signal.

2. Thresholding adjustment of wavelet coefficients.

3. Signal reconstruction via L-Level Inverse Wavelet Transform.

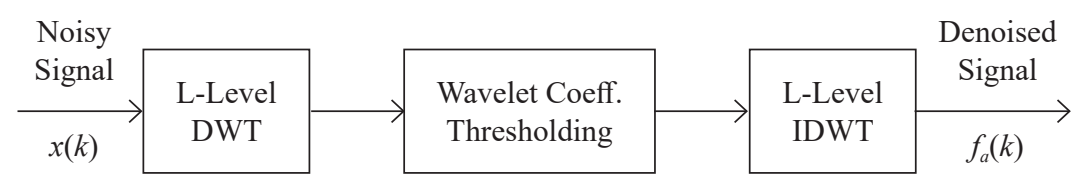

Figure 3: De-noising scheme.

Here, the thresholding selection rule consists of four rules which is the principle of Stein's Unbiased Risk Estimate (rigrsure), heuristic variant (heursure), universal threshold (sqtwolog) and minimax thresholding (minimaxi). 


\subsection{Heartbeat Segmentation}

In this research, we use the detection of $R$ peak as our reference in segmentation. The selection of appropriate wavelet and the number of decomposition level contribute greatly in the analysis of signal. ${ }^{15}$ The raw signals are first decomposed using $d b 6$ and the number of decomposition level is eight. Therefore, the signals are decomposed into the details $d_{1}-d_{8}$ and one approximation $a_{8}$. Frequency components at detail levels $d_{3}-d_{7}$ represent the useful part of the heartbeat while other details level contains unwanted noise. The steps of segmenting the useful part of ECG from the raw ECG signal are as follow:

1. Applying wavelet de-noising on the raw ECG signal.

2. Finding means for peak height and peak distance.

3. Applying a window of 500 discrete points on each raw signal which has 10,000 discrete points.

4. Detecting $R$ peak locations by using the means for peak height and peak distance found.

5. Moving to another window if there is no peak detected.

6. Using $R$ peak location as a reference point, a segment is taken around the $R$ peak to cover the useful part of ECG.

\subsection{Feature Extraction: Types of Mother Wavelets}

As discussed, wavelet transformation is employed for feature extraction. The heartbeat signals contain useful features, futile features and also noises. This study uses fiducial points from ECG signal to extract features. In this case, the fiducial points are the $R$ peaks in ECG. The $R$ peak locations found during segmentation stage will be used as a reference for extracting the heartbeat. The steps to obtain useful features from the ECG are as follow:

1. Applying wavelet de-noising on the raw signals.

2. With the peak locations found during segmentation, the heartbeat is segmented.

3. Applying wavelet decomposition to the heartbeat into eight levels by using $d b 6$ wavelet.

4. Thresholding the low-level details to 0 .

5. Applying wavelet reconstruction to the thresholded signal.

6. Signal normalisation. 
7. Concatenating the significant wavelet coefficients in a $1 \mathrm{D}$ array.

8. Repeat the feature extraction process for other two different mother wavelets, which are sym 4 and coif 2 to produce three different sets of features.

\subsection{Classification}

In classification stage, there are two steps involved: training and testing. In training phase, one recording from each subject which makes a total of 79 samples is used as the training samples. Based on the models constructed by the Support Vector Machine (SVM), the testing data are verified based on the set threshold $(T S)$. When the score exceeds the Ts, the person is recognised an authentic user otherwise as an imposter. The Kernel function used for the SVM training is the Gaussian Radial Basis Function (rbf) with the scaling factor, $\sigma$ of 4.

\subsection{ECG Based Security System}

A simple security system is developed for testing the feasibility of the heartbeat biometric algorithm. In this system, the processes include detecting ECG signal from the subjects, transmitting the signal to the server via the internet, data processing using the developed biometric system and lastly user authentication. The working flow of the system is shown in Figure 4. Using a mobile device, the user is required to login an Android application using identification (ID). The user is asked to upload his ECG data for security check. The mobile device collects the heartbeat signal from the user and sends the data to the cloud server through a Wi-Fi connection. The heartbeat signal will be downloaded from the server to be processed by the developed biometric system. The permission to unlock the application is granted for the genuine user. On the other hand, imposter user is denied.

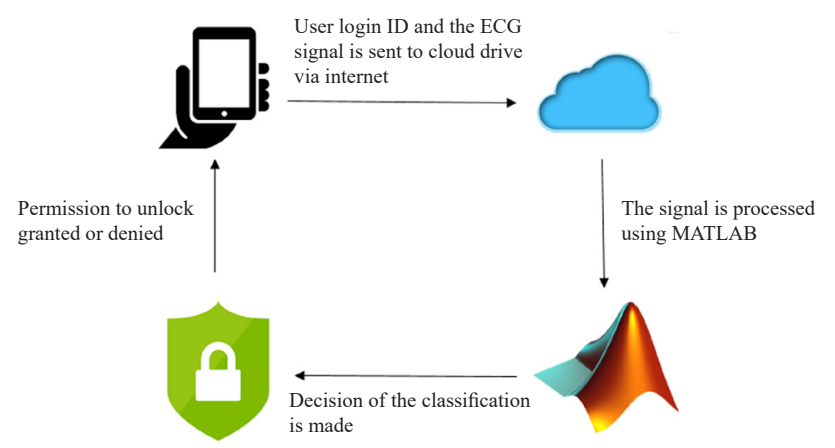

Figure 4: Working flow of the proposed security system. 

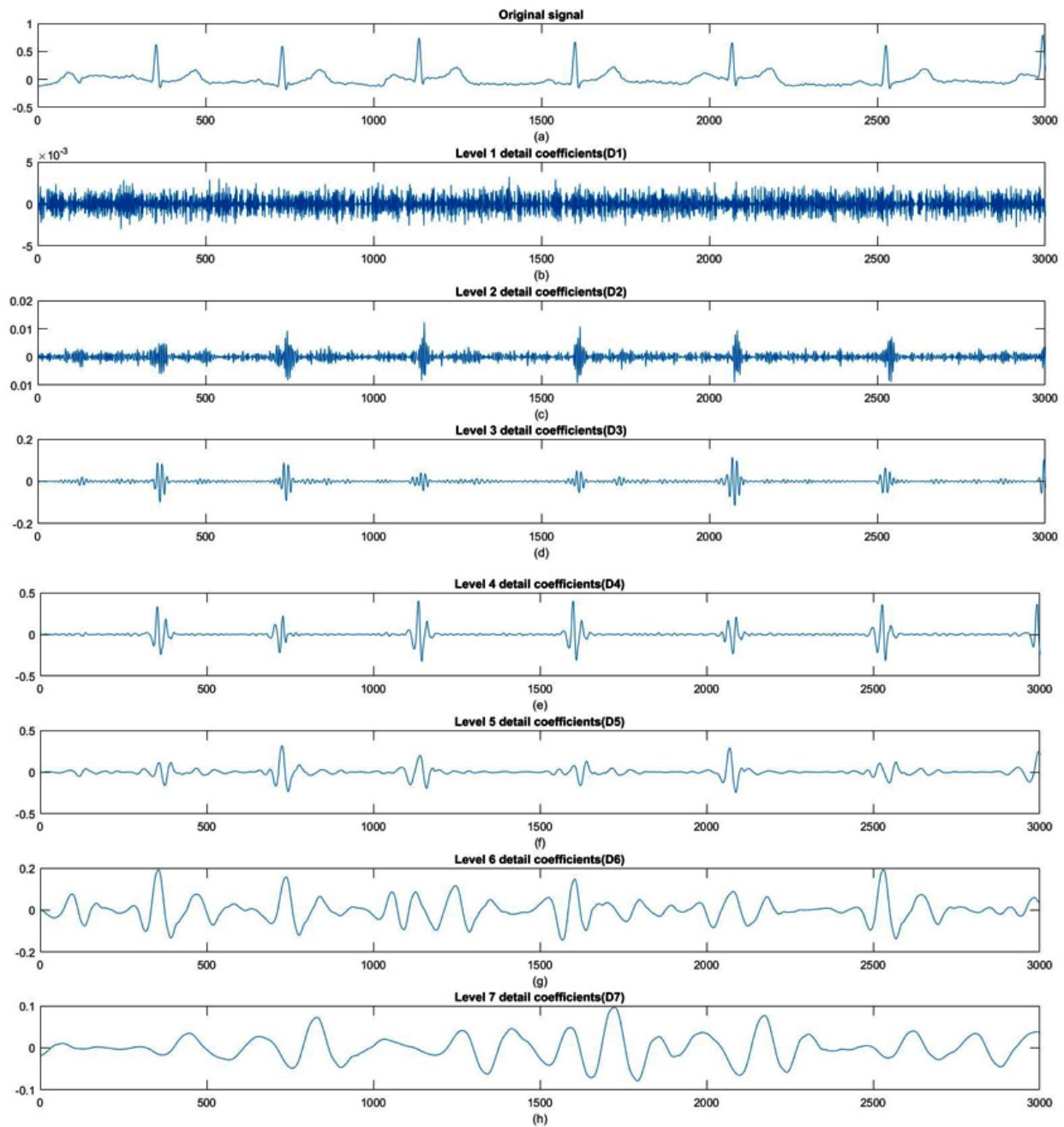

(h)
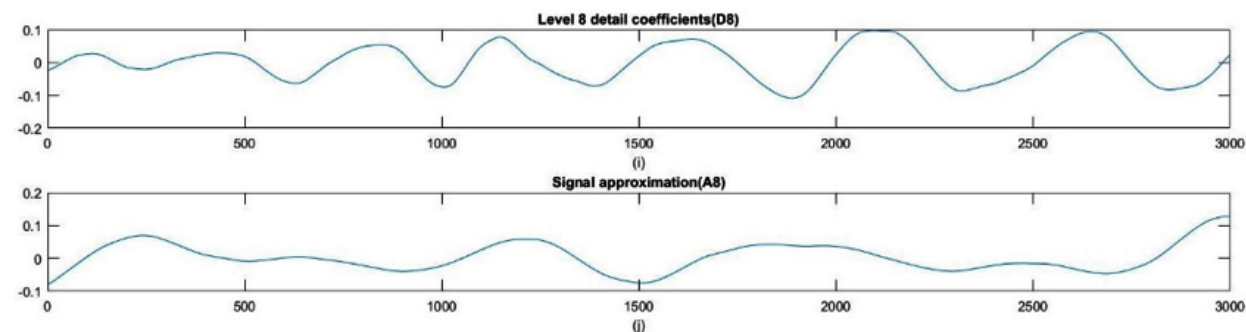

Figure 5: (a) Original signal, (b-i) wavelet decompositions for levels 1 to 8 respectively and (j) signal approximation. 


\section{RESULT AND DISCUSSION}

\subsection{Pre-processing}

The original signal is shown in Figure 5(a). The original signal was decomposed in eight levels using $d b 6$ wavelets. From Figure 5, it is observed that the baseline shifting is most concentrated at scale 8 . The low frequency components D8 and A8 were removed to obtain a baseline-free signal.

The original signal, baseline-removed signal and de-noised signal are shown in Figure 6,7 and 8. Thus, noise still presents in the de-trended signal due to the high frequency components, the components of D1 and D2 were filtered called as de-noised signal.

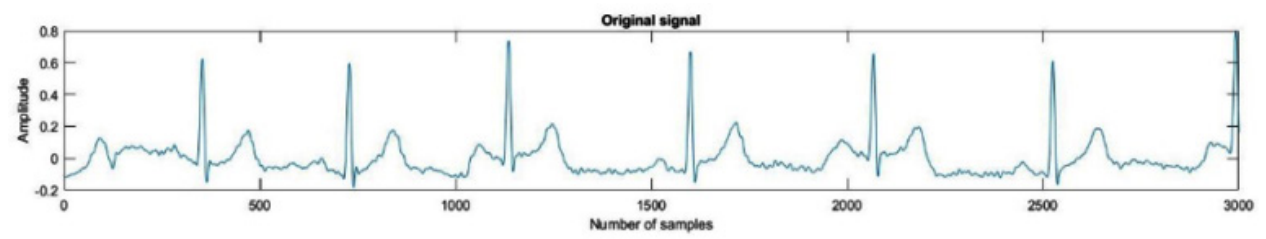

Figure 6: Original signal.

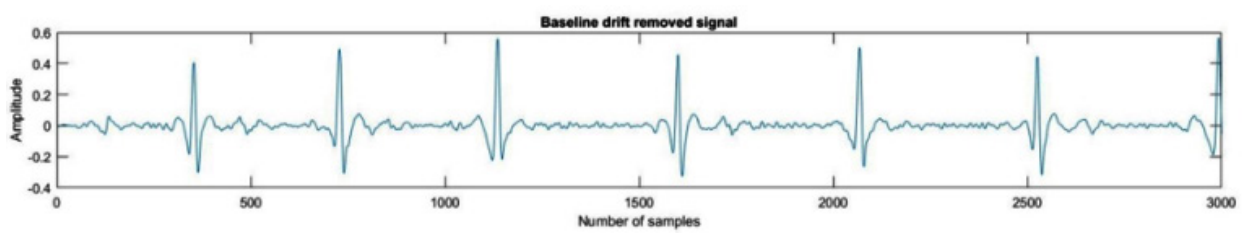

Figure 7: Baseline drift removed signal.

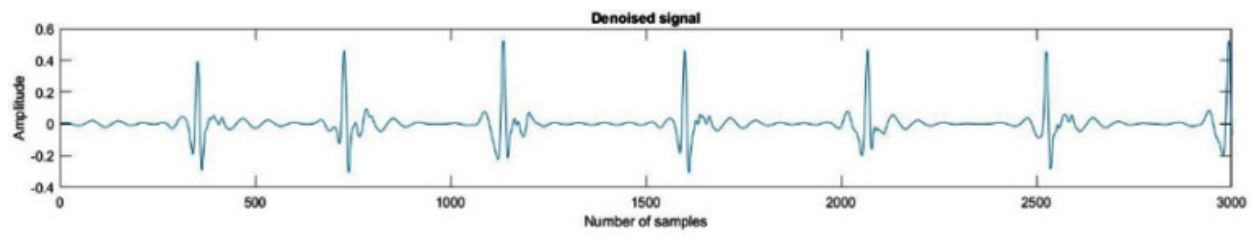

Figure 8: De-noised signal. 


\subsection{Heartbeat Segmentation}

The segmentation of heartbeat includes the QRS complex as it is the essential feature for the classification algorithm. As shown in Figure 9, the highlighted parts are the segmented heartbeats. By using the locations of $R$ peaks detected, the heartbeat is segmented by taking 70 data points to the left of the $R$ peak and 50 data points to the right of $R$ peak.

\subsection{Feature Extraction}

The comparison of the segmented ECG signals from two individuals taken from the database which is Person A and Person B are shown in Figure 10 and 11 , respectively. Both individual's segmented ECG signals look similar however with detail observation, the amplitude of $R$ is different and the $T$ wave occurs at a different time. The wavelet coefficients of Person A and Person B obtained after the feature extraction process are then shown in Figure 12 and 13, respectively. By observing the coefficient from both individuals, the patterns are clearly unique from one another.

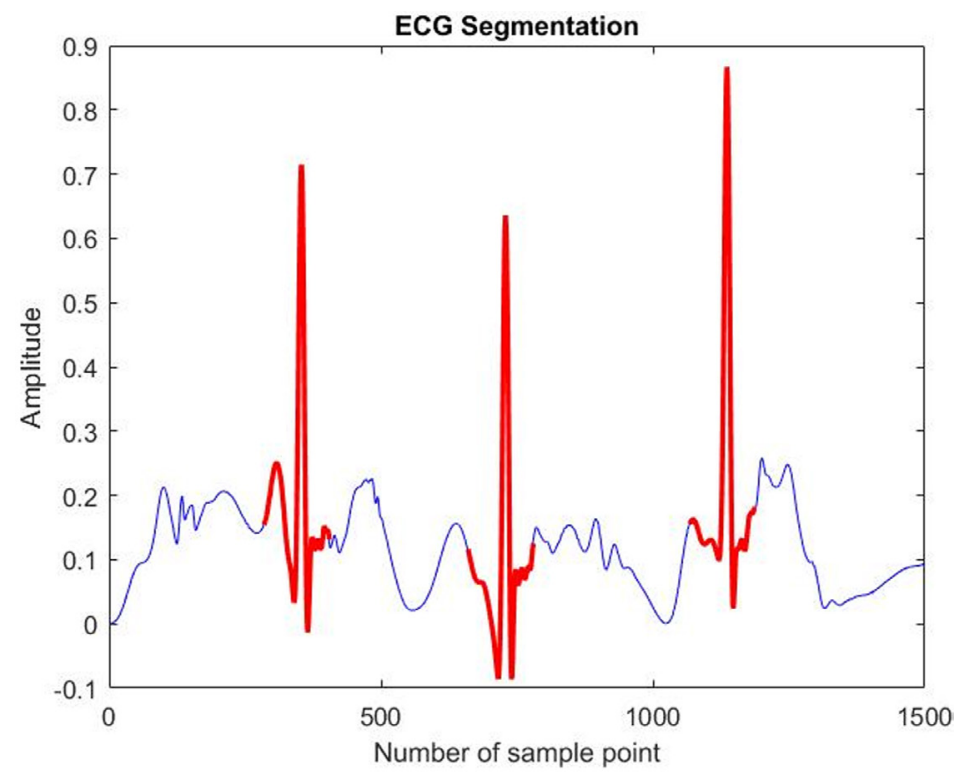

Figure 9: ECG segmentation. 


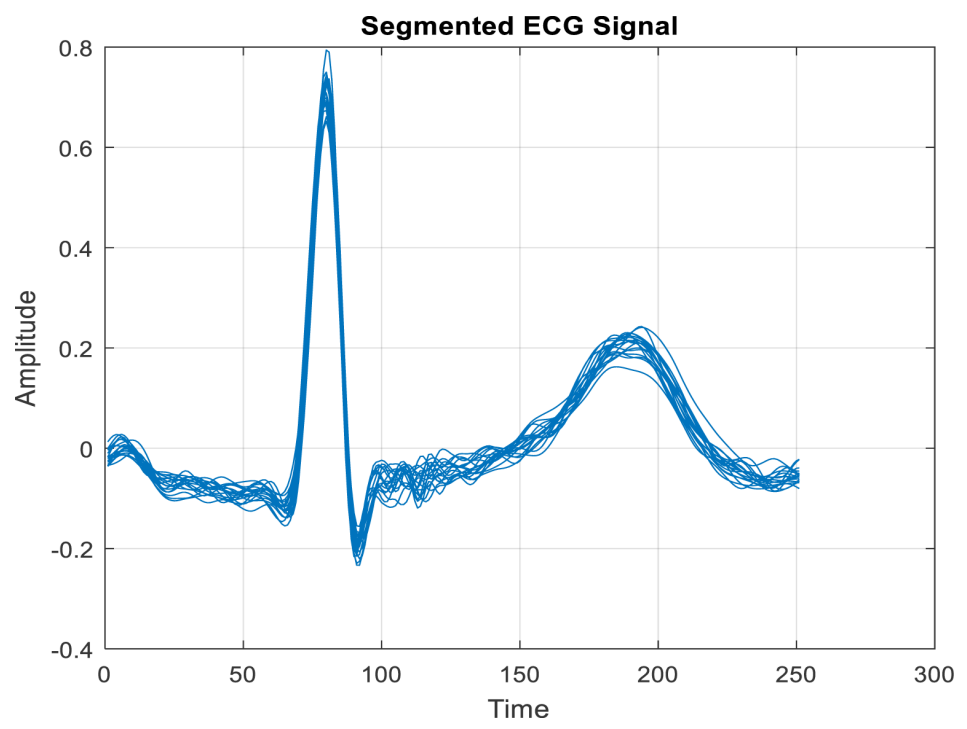

Figure 10: Heartbeats obtained for individual A.

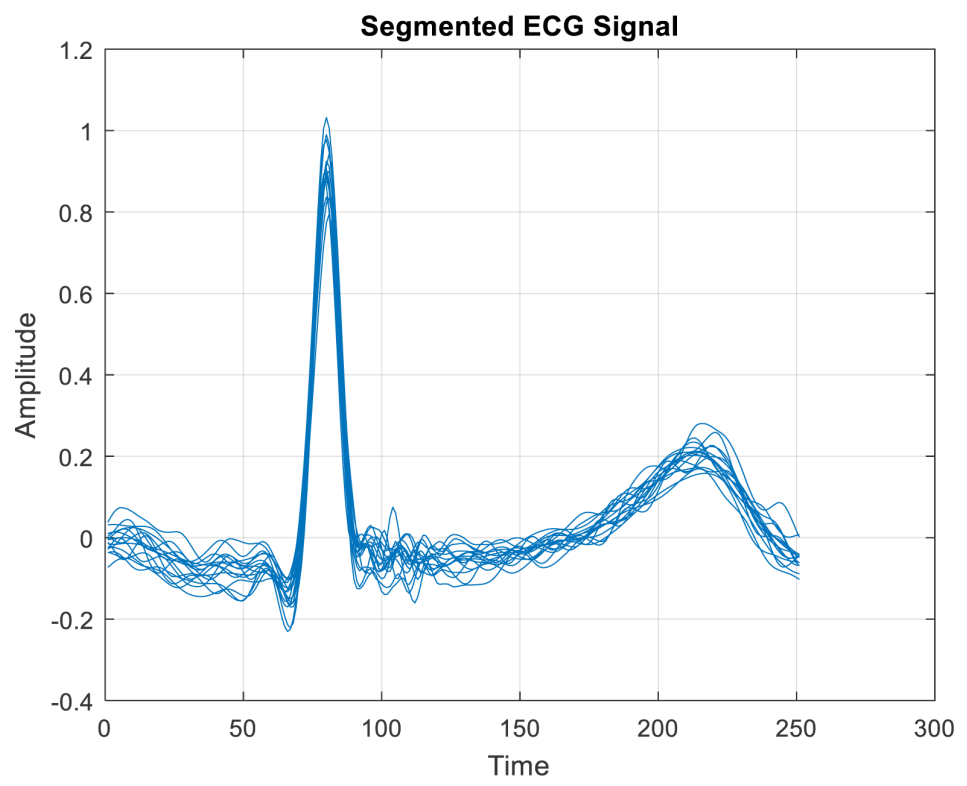

Figure 11: Heartbeats obtained for individual B. 


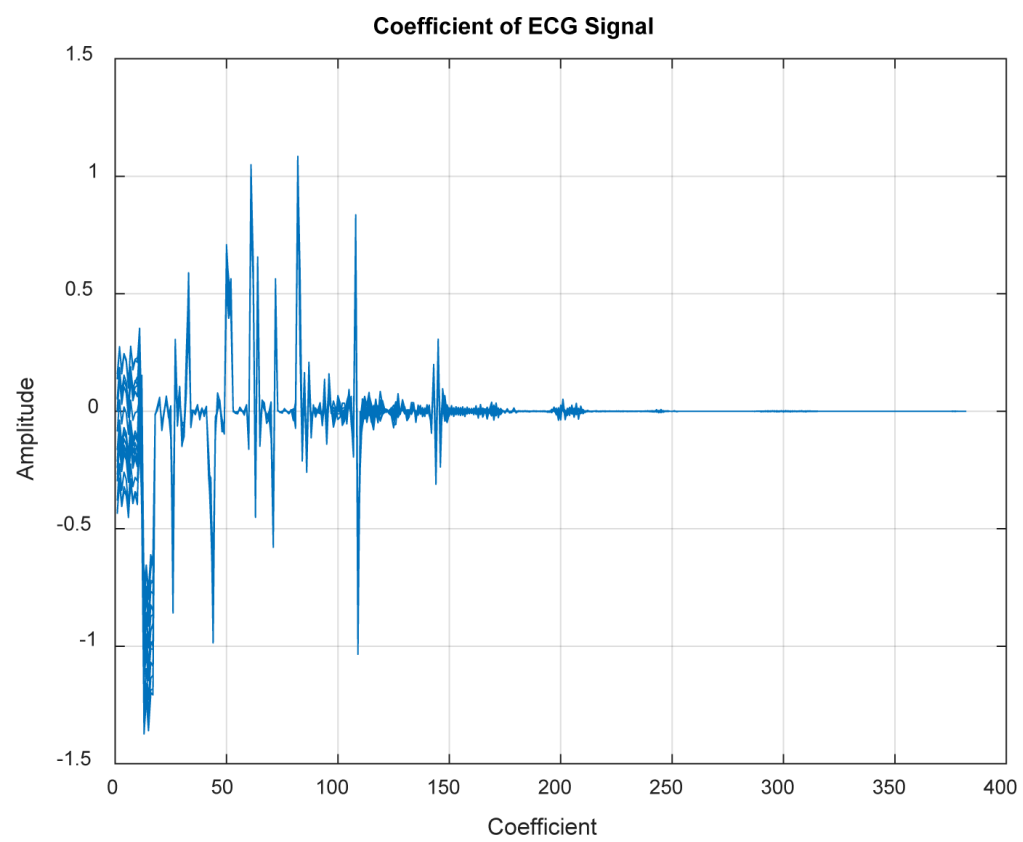

Figure 12: Wavelet coefficients for individual A.

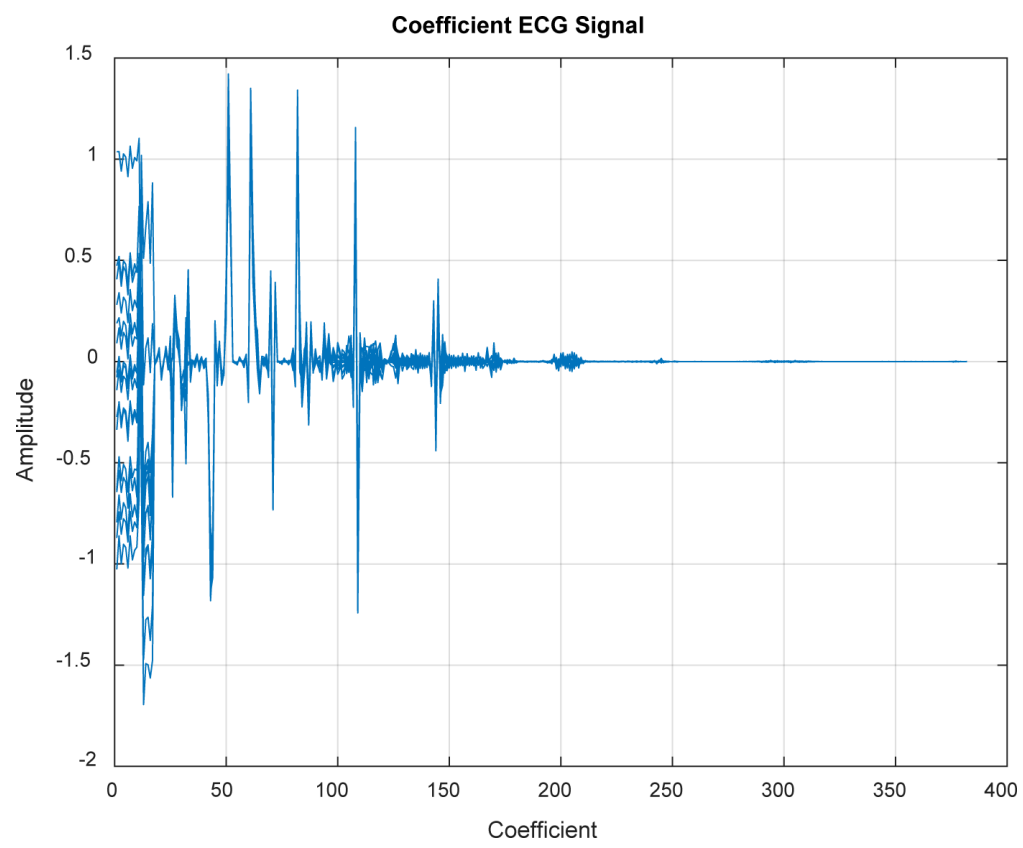

Figure 13: Wavelet coefficients for individual B. 


\subsection{Evaluation of the System Performance}

A comparison of the performance of the system was performed by varying different parameters including wavelet de-noising order, wavelet coefficient threshold method and types of mother wavelet. The accuracy is based on the evaluation of the receiver operating characteristic (ROC) which is a plot of the Genuine Acceptance Rate (GAR) against the False Acceptance Rate (FAR) at various threshold settings. Equal Error Rate (EER) is also used to analyse the system performance. Figure 14 shows that the system with de-noising order 3 has the best performance with GAR of $91.5 \%$ at FAR of $5 \%$, and the EER of $7.9398 \%$. The system performance decreased as the de-noising order increased. The overall performances in term of EER are given in Table 1.

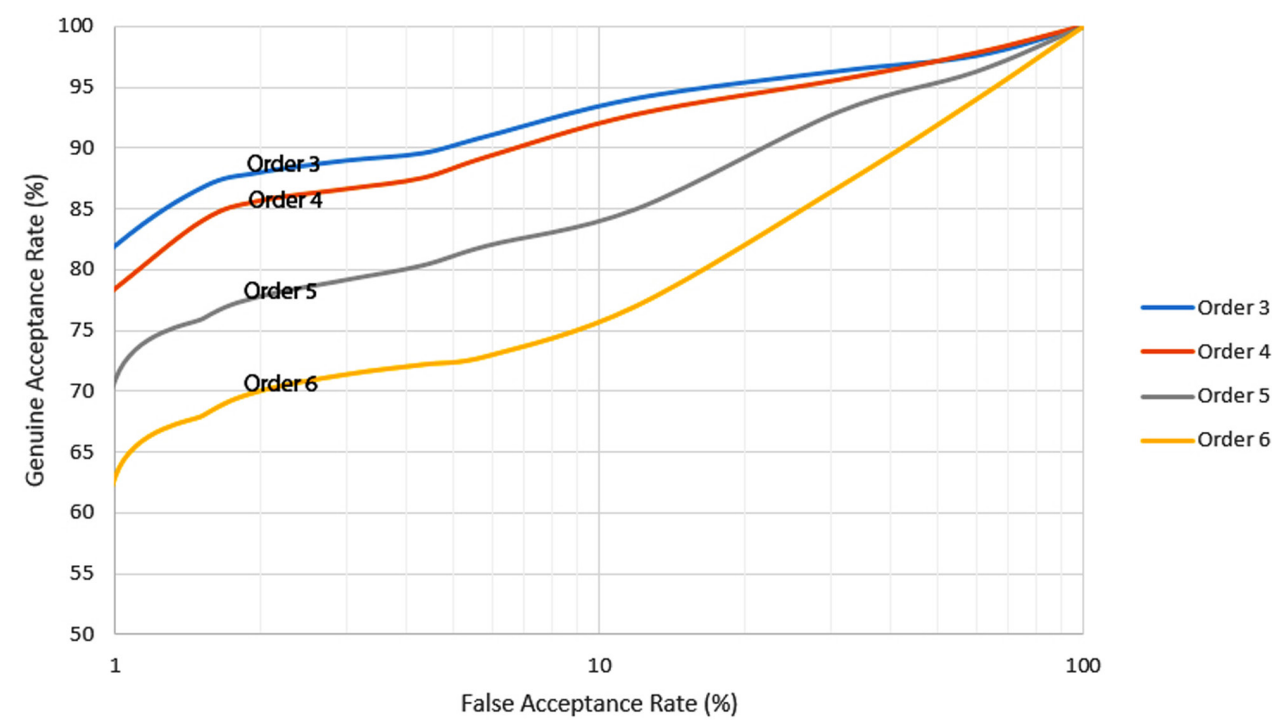

Figure 14: ROC curve for different de-noising orders.

Table 1: EER performance for different wavelet de-noising orders.

\begin{tabular}{cc}
\hline Wavelet de-noising order & EER (\%) \\
\hline 3 & 7.9398 \\
4 & 8.5382 \\
5 & 13.5751 \\
6 & 18.9813 \\
\hline
\end{tabular}


The four wavelet coefficient threshold methods that employed are heursure, minimaxi, rigrsure and sqtwolog. heursure threshold method achieved the best performance with GAR of $92 \%$ at FAR of $5 \%$ as shown in Figure 15 . The overall performances in term of EER are given in Table 2.

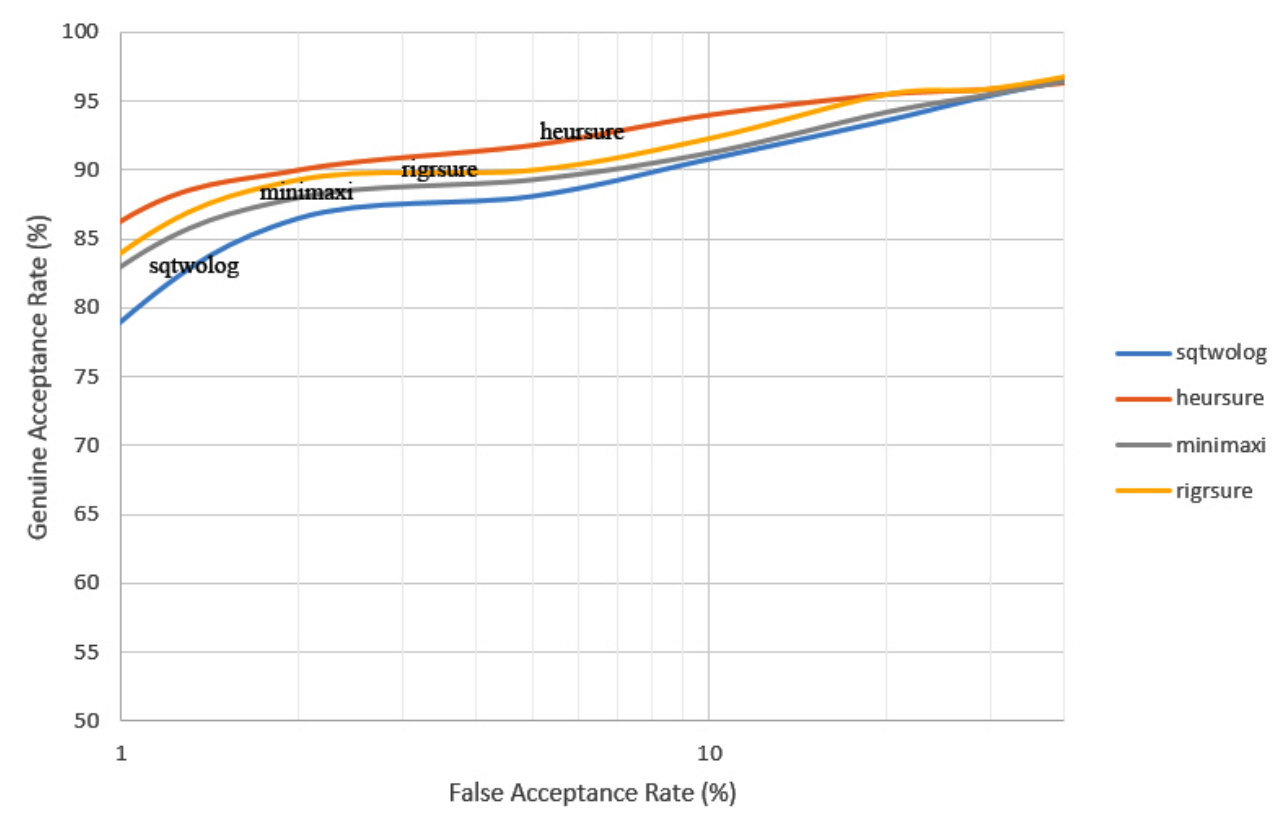

Figure 15: ROC curve for different threshold methods.

Table 2: EER performance for different wavelets threshold methods.

\begin{tabular}{cc}
\hline Threshold method & EER $(\%)$ \\
\hline heursure & 6.9600 \\
rigrsure & 7.9438 \\
sqtwolog & 8.5382 \\
minimaxi & 8.8973 \\
\hline
\end{tabular}

The types of mother wavelet implemented in this project were $d b 6$, sym 4 and coif 2 . From Figure 16, the system performances for the three mother wavelets were very close to each other. System with $d b 6$ achieved the highest GAR of $89 \%$ and the lowest ERR of $8.5382 \%$. The lowest performance system was sym 4 with GAR of $87 \%$ at FAR of $5 \%$, with $2 \%$ GAR lower than of $d b 6$. The overall performances in term of EER are given in Table 3. 


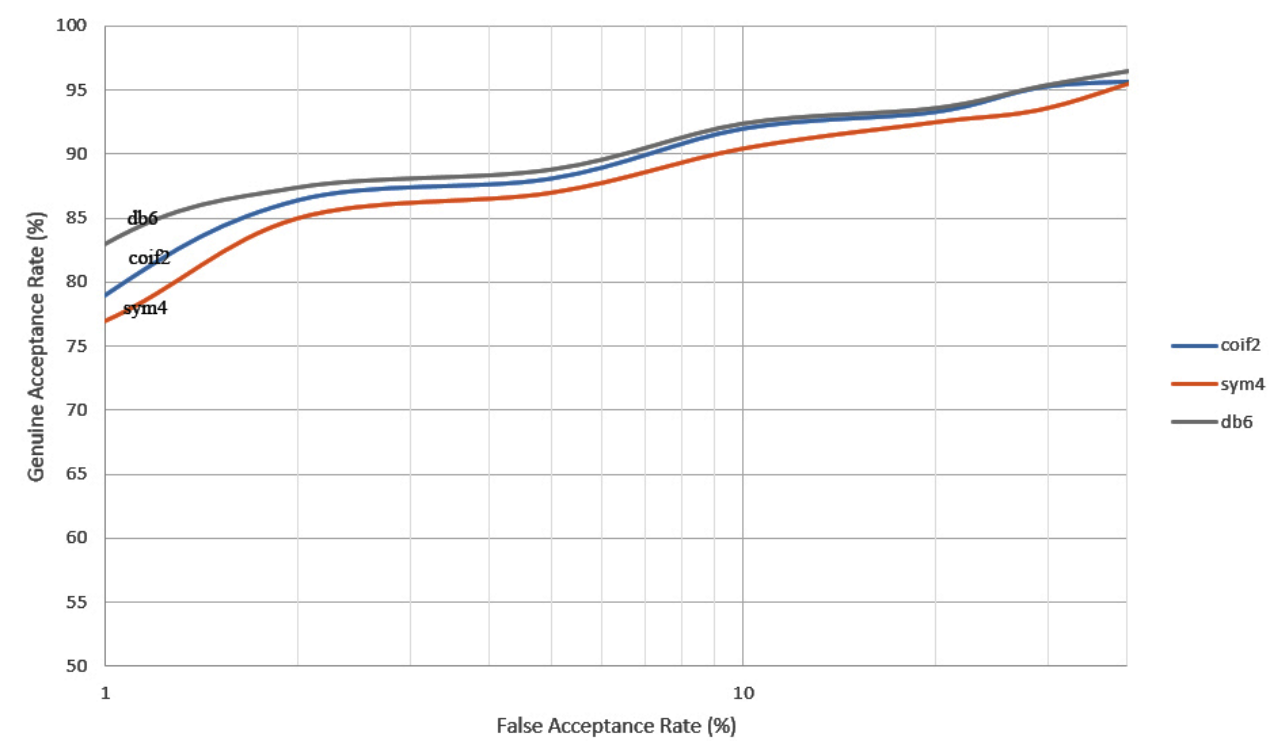

Figure 16: ROC curve for different mother wavelets.

Table 3: EER performance for different types of mother wavelets.

\begin{tabular}{cc}
\hline Type of mother wavelet & EER (\%) \\
\hline db6 & 8.5382 \\
coif2 & 9.1894 \\
sym4 & 9.5809 \\
\hline
\end{tabular}

Consequently, system with different combinations of wavelet de-noising order, threshold method and mother wavelet is then developed and their system performance is tested. The system with the combination of de-noising order 3 , heursure threshold method and $d b 6$ mother wavelet produced the best result with GAR of $92.5 \%$ at FAR of $5 \%$ and EER of $6.9499 \%$.

Finally, the security system is developed by setting one of the subjects in the database (namely "Person1") as the genuine user and the Ts for accepting or rejecting the claimed user is set as 0.5 . The user was required to upload the ECG data via the mobile phone and claimed as "Person1". When "SELECT DATA" is clicked, the user can browse the mobile phone storage and upload the Person1's ECG data to the cloud server. The data will be downloaded to the laptop and processed by developed biometric system. Clicking the button "START" to authenticate the user, the score generated by the system was 0.82 . As recognised as the genuine user (as the score is greater than Ts which is equal to 0.5 ), the login 
is successful and this genuine user is granted a permission to switch on and switch off the "Control1" and "Control2" as shown in Figure 17. The system recognised the user as imposter user when "Person2" data was uploaded but claiming as "Person $1 "$. The score generated for the user was 0.13 and the user was denied to login the application.

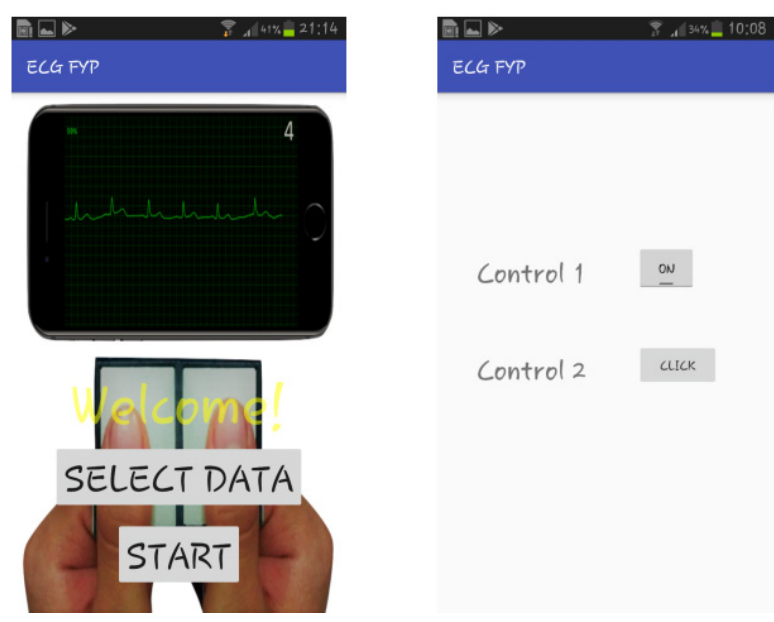

Figure 17: Main interface and control interface.

\section{CONCLUSION}

This study shows that the developed heartbeat based biometric system using discrete wavelet transform and support vector machine achieves satisfying accuracy for individual authentication under normal heart rate condition. The combination of de-noising order 3, heursure threshold method and $d b 6$ mother wavelet yielded the top performance. The developed authentication process is successfully implemented in a security system. The feasibility of the developed system can be further improved by developing an algorithm which is able to recognise ECG under different physical and physiological conditions. Further analysis and experiments are needed to be carried out to evaluate the system with large database that comprises subjects of different ages, genders, races and abnormal ECG data.

\section{ACKNOWLEDGEMENTS}

This research was supported by Universiti Sains Malaysia under the Fundamental Research Grant Scheme (6071266). 


\section{REFERENCES}

1. Rattani, A. (2015). Introduction to adaptive biometric systems. In Rattani, A., Roli, F. \& Granger, E. (eds.), Advance in computer vision and pattern recognition. Olten, Switzerland: Springer, 1-8, https://doi. org/10.1007/978-3-319-24865-3 1.

2. O'Gorman, L. (2003). Comparing passwords, tokens, and biometrics for user authentication. Proc. IEEE, 91(12), 2021-2040, https://doi. org/10.1109/JPROC.2003.819611.

3. Hedge, C. et al. (2011). Heartbeat biometrics for human authentication. Signal Image Video P., 5(4), 485-493, https://doi.org/10.1007/s11760011-0252-6.

4. Chuang-Chien, C., Chou-Min, C. \& Chih-Yu, H. (2008). A novel personal identity verification approach using a discrete wavelet transform of the ECG signal. Paper presented at the International Conference on Multimedia and Ubiquitous Engineering. Busan, South Korea, 24-26 April.

5. Medical Training and Simulation LLC. (2017). ECG, EKG, heart sounds, murmurs, lung sounds. In Practical clinical skills. Retrieved from https://www.practicalclinicalskills.com/cardiac-rhythms on 10 June 2017.

6. Sörnmo, L. \& Laguna, P. (2005). Bioelectrical signal processing in cardiac and neurological applications, biometric engineering. Cambridge, MA: Elsevier Academic Press.

7. Malmivuo, J. \& Plonsey, R. (1995). Bioelectromagnetism: Principles and applications of bioelectric and biomagnetic fields. New York: Oxford University Press, https://doi.org/10.1093/ acprof:oso/9780195058239.001.0001.

8. Kyoso, M. \& Uchiyama, A. (2001). Development of an ECG identification system. In Conference proceedings of the 23rd annual international conference of the IEEE Engineering in Medicine and Biology Society. Piscataway, NJ: Institute of Electrical and Electronics Engineers, Inc. (IEEE), https://doi.org/10.1109/IEMBS.2001.1019645.

9. Plataniotis, K. N., Hatzinakos, D. \& Lee, J. K. M. (2006). ECG biometric recognition without fiducial detection. In 2006 Biometrics symposium: Special session on research at the biometric consortium conference. Piscataway, NJ: Institute of Electrical and Electronics Engineers, Inc. (IEEE), https://doi.org/10.1109/BCC.2006.4341628.

10. Saechia, S., Koseeyaporn, J. \& Wardkein, P. (2005). Human identification system based ECG signal. Paper presented at the Proceedings of IEEE TENCON Region 10. Bangkok, Thailand, 5-8 November, https://doi. org/10.1109/TENCON.2005.300986. 
11. Belgacem, N. et al. (2012). ECG based human authentication using wavelets and random forests. IJCIS, 2(2), 1-11, https://doi.org/10.5121/ ijcis.2012.2201.

12. Ramli, D. A., Hooi, M. Y. \& Chee, K. J. (2016). Development of heartbeat detection kit for biometric verification system. Procedia Computer Science, 96, 305-314, https://doi.org/10.1016/j.procs.2016.08.143.

13. Goldberger, A. L. et al. (2000). PhysioBank, PhysioToolkit, and PhysioNet: Components of a new research resource for complex physiologic signals. Circulation, 101(23), 215-220, https://doi.org/10.1161/01.CIR. 101.23.e215.

14. The MathWorks, Inc. (n.d.). MATLAB \& Simulink. Documentation: Wavelet denoising. Massachusetts: The MathWorks, Inc. Retrieved from https://www.mathworks on 3 June 2017.

15. Zhao, Q. \& Zhang, L. (2005). ECG feature extraction and classification using wavelet transform and support vector machines. Paper presented at the International Conference on Neural Networks and Brain. Beijing, China, 13-15 October. 